\title{
ANÁLISIS COMPUTACIONAL DE LESIONES CERVICALES NO CARIOSAS EN UN PREMOLAR SUPERIOR
}

\section{COMPUTER ANALYSIS OF NON-CAVITY CERVICAL LESIONS IN AN UPPER PREMOLAR}

\author{
Patricio Cendoya $^{1} \quad$ Jorge Hernández $^{2} \quad$ Emilio Dufeu $^{1}$ \\ Recibido 20 de noviembre de 2006, aceptado 12 de julio de 2007 \\ Received: November 20, 2006 Accepted: July 12, 2007
}

\begin{abstract}
RESUMEN
Se presenta un modelo tridimensional de elementos finitos para investigar el efecto producido por las fuerzas de oclusión en la distribución y magnitud del campo tensional sobre un premolar superior. De esta forma, se busca definir cuál es la situación de carga de oclusión más crítica desde el punto de vista tensional sobre el esmalte dental en la zona cervical que pudiese dar origen a una lesión del tipo no cariosa. Utilizando elementos finitos hexaédricos lineales y procesando el modelo con el programa SAMCEF se realiza un análisis numérico estático lineal considerando que el premolar es isótropo y homogéneo. Se definen siete estados de carga asociados a una fuerza de oclusión de $170 \mathrm{~N}$ para las cuales se investiga la distribución y magnitud de los campos tensionales sobre el premolar. Los resultados numéricos permiten concluir que las tensiones máximas sobre el premolar tienden a concentrarse en la zona cervical alcanzando sus valores máximos cuando la carga de $170 \mathrm{~N}$ actúa de forma inclinada a $45^{\circ}$ con respecto a la vertical. La relevancia de la presente investigación radica en el hecho que numéricamente se demuestra que la distribución y zona en donde los campos tensionales tienden a concentrase y alcanzar sus valores máximos son coincidentes con las zonas del diente en donde clínicamente se observan lesiones no cariosas.
\end{abstract}

Palabras clave: Lesiones cervicales no cariosas, método elementos finitos, biomecánica dental.

\section{ABSTRACT}

A three-dimensional model of finite elements in order to investigate the effect produced by occlusion forces on both distribution and magnitude of the tensional field on an upper premolar is hereby presented. Thus, defining from a tensional point of view, which is the situation of the most critical occlusion loading on dental enamel capable to produce a non-cavity lesion is intended. By using finite linear hexahedral elements and processing the models through SAMCEF software, a static linear numerical analysis is performed, considering that premolar is isotropic and homogeneous. Seven load states associated to occlusion force of $170 \mathrm{~N}$ are defined and the distribution and magnitude of tensional fields on premolar are also investigated. Numerical results allow concluding that maximum tensions on premolar tend to concentrate around cervical area, reaching their maximum values when the $170 \mathrm{~N}$ load acts inclined $45^{\circ}$ from vertical. Relevance of current research lies on the fact that both distribution and area where tensional fields tend to concentrate and reach maximum values has been numerically proved. These values are coincident with tooth areas where non-cavity lesions are clinically observed.

Keywords: Non cavity cervical lesions, finite element methods, dental biomechanics.

\section{INTRODUCCIÓN}

Un diente está compuesto por tres tejidos duros: esmalte, dentina y cemento y uno blando llamado pulpa. El esmalte es el tejido periférico ubicado en toda la porción libre o visible del diente (corona), siendo éste más grueso en la cara oclusal y más delgado en la zona cervical. Interiormente se ubica la dentina, la cual circunscribe una porción ocupada

\footnotetext{
1 Facultad de Ingeniería. Universidad de Concepción. Casilla 160-C. Correo 3. Concepción, Chile. E-mail: pcendoya@udec.cl, edufeu@udec.cl

2 Facultad de Odontología. Universidad de Concepción. Casilla 160-C. Correo 3. Concepción, Chile. E-mail: jhernand@udec.cl
} 
por la pulpa (tejido blando), rodeando toda la superficie radicular se ubica el cemento. Las lesiones cervicales no-cariosas se definen como una pérdida del tejido dental duro del diente (esmalte); generalmente estas lesiones se producen donde se inicia la unión cemento-esmalte, es decir, en la zona cervical. Clínicamente, las lesiones cervicales son defectos de contorno que se desarrollan principalmente en la zona labial o bucal del diente. Las causas de lesiones cervicales no-cariosas es fuente de gran controversia en la comunidad odontológica, ya que existen múltiples y diferentes teorías [6]. Sin embargo, en los últimos años se ha postulado que las tensiones producidas por las fuerzas de oclusión sobre los dientes son la causa del desarrollo de las lesiones cervicales no-cariosas. Esta teoría, denominada de abfracción por Grippo [3] en base al trabajo previo de Lee y Eackle [4], plantea que las fuerzas de oclusión lateral generadas durante la masticación, parafunciones (bruxismo) y oclusión desbalanceadas hacen que el diente se flexione y se generen esfuerzos de tracción y compresión. Los esfuerzos de tracción tienden a concentrarse en la zona cervical y pueden hacer que los prismas de hidroxiapatita que componen el esmalte se quiebren y pueda producirse la separación entre ellos. Sucedido esto, pequeñas partículas y líquido pueden penetrar los prismas de hidroxiapatita rotos y hacer el diente más susceptible a la erosión química y a la abrasión por cepillado.

En este contexto y a través del empleo de modelos de elementos finitos, diversos investigadores han estudiado lesiones cervicales [2, 5 y 7] sobre diversas piezas dentarias y han obtenido conclusiones acerca del comportamiento biomecánico de dichas piezas. El presente artículo aborda el problema de encontrar la distribución y magnitud del campo tensional sobre un premolar superior cuando éste se somete a niveles de fuerzas de oclusión que simulan el proceso de masticación. Se busca correlacionar los resultados numéricos con las hipótesis de la teoría de abfracción [3] y las observaciones clínicas, de forma de comprender desde un punto de vista mecánico el mecanismo de inicio de la lesión cervical no cariosa.

\section{MATERIAL Y MÉTODOS}

Discretización espacial: a partir del modelo geométrico de un premolar superior sano, se genera una discretización de elementos finitos hexaédricos que considera tres tejidos de la corona del diente (esmalte, dentina y pulpa). En una primera etapa, el dominio acotado por la superficie del premolar se divide en subdominios de menor tamaño y se procede a la generación automática de elementos hexaédricos en cada uno de ellos. En una segunda etapa, la discretización se interviene manualmente para mejorar la razón de aspecto de algunos elementos. Finalmente se obtiene una malla de elementos finitos constituida por 9207 nodos y 27621 grados de libertad, ver figura 1 [1]. Puesto que las lesiones cervicales afectan principalmente a los tejidos duros de la corona (esmalte y dentina), el modelo de elementos finitos no considera en la discretización al cemento que rodea la raíz del premolar superior.

Propiedades mecánicas: las propiedades mecánicas de los materiales constituyentes de la estructura del diente se resumen en la tabla 1. El presente estudio considera que los materiales constituyentes del premolar (esmalte, dentina y pulpa) presentan un comportamiento elástico lineal y que cada uno de ellos ocupa regiones en el espacio en donde la estructura interna es homogénea e isótropa.

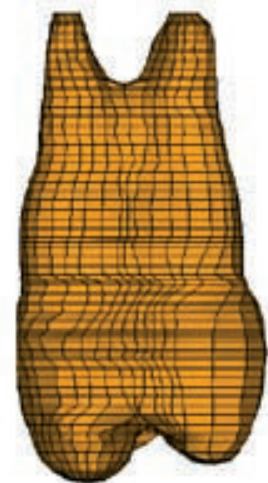

(a)

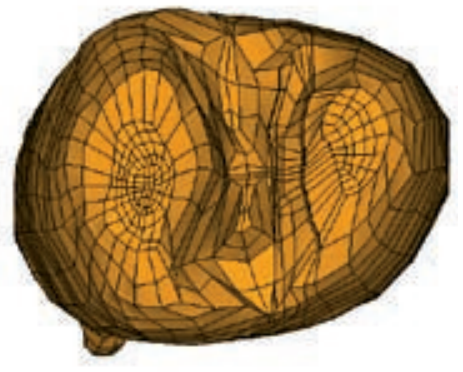

(b)
Figura 1. Vista discretización elementos finitos hexaédricos. a) Cara mesial, b) Cara oclusal.

Tabla 1. Propiedades elásticas de los tejidos utilizados en el estudio.

\begin{tabular}{|l|c|c|}
\hline \multicolumn{1}{|c|}{ Tejido [Ref.] } & $\begin{array}{c}\text { Módulo de } \\
\text { Young (MPa) }\end{array}$ & $\begin{array}{c}\text { Módulo } \\
\text { de Poisson }\end{array}$ \\
\hline Esmalte [2] & 84,100 & 0,20 \\
\hline Dentina [5] & 18,600 & 0,31 \\
\hline Pulpa [9] & 2 & 0,45 \\
\hline
\end{tabular}

Condiciones de contorno: el premolar se encuentra impedido de desplazarse en su zona inferior (raíz), debido a que en dicho lugar se encuentra en contacto con el hueso maxilar. Para representar dicha condición 
se fijan e imponen igual a cero los desplazamientos en las direcciones de los ejes X, Y y Z en la zona de contacto del premolar superior con el hueso maxilar.

Estados de carga: se analizan por separado siete estados de carga asociados al proceso de masticación tal como se indica en la figura 2.

1. E_1 (estado de carga 1): Carga vertical en la cúspide lingual.

2. E_2 (estado de carga 2): Carga bucal a $45^{\circ}$ del eje vertical en la cúspide lingual.

3. E_3 (estado de carga 3): Carga lingual a $45^{\circ}$ del eje vertical en la cúspide lingual.

4. E_4 (estado de carga 4): Carga vertical en la fosa central.

5. E_5 (estado de carga 5): Carga vertical en la cúspide bucal.

6. E_6 (estado de carga 6): Carga bucal a $45^{\circ}$ del eje vertical en la cúspide bucal.

7. E_7 (estado de carga 7): Carga lingual a $45^{\circ}$ del eje vertical en la cúspide bucal.

La magnitud de la carga estática es de $170 \mathrm{~N}$ [5] y se distribuye localmente en la zona de aplicación.

Análisis estructural: se realiza con el programa de elementos finitos SAMCEF V11.1 [8] considerando que las cargas se aplican en forma estática.

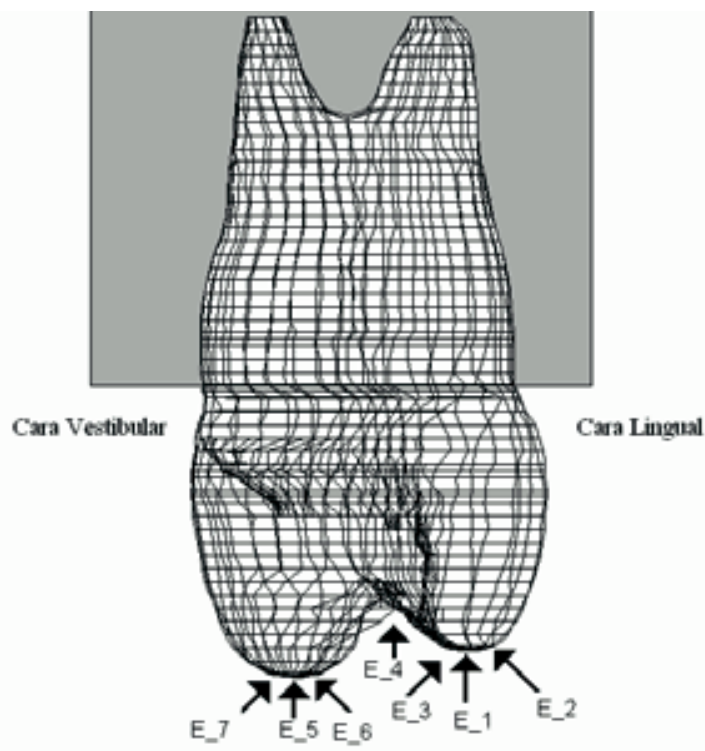

Figura 2. Cara mesial con sus respectivos estados independientes de carga.

\section{RESULTADOS}

Los resultados numéricos indican que las tensiones principales máximas tienden a concentrarse en la zona cervical de la cara mesial del lado vestibular del premolar. Para comprender la evolución de dichas tensiones sobre el esmalte dental, se grafican sus magnitudes sobre una recta $\mathrm{AB}$ que atraviesa el premolar desde la cara lingual hacia la vestibular, ver figura 3.

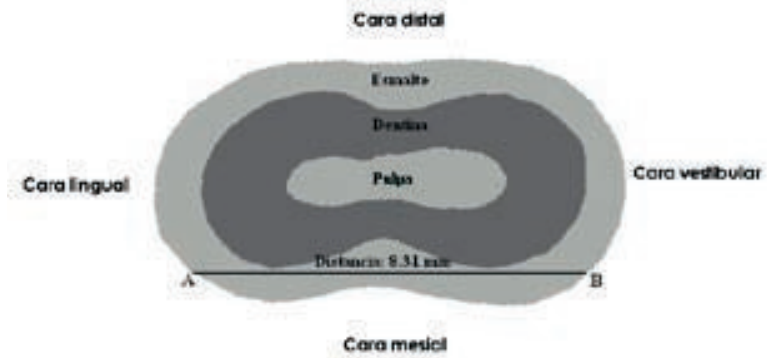

Figura 3. Sección transversal del premolar superior a nivel cervical.

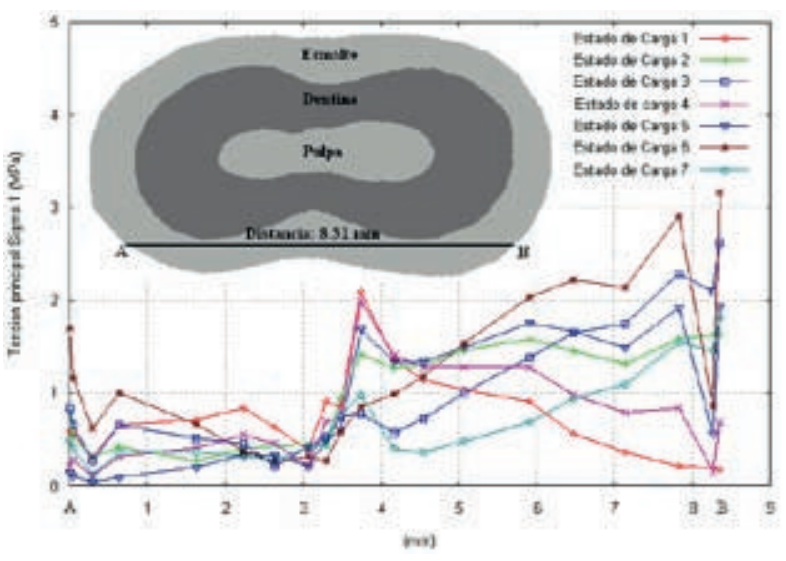

Figura 4. Distribución de tensión principal $\sigma_{1}$ (tracción) a lo largo de la distancia AB.

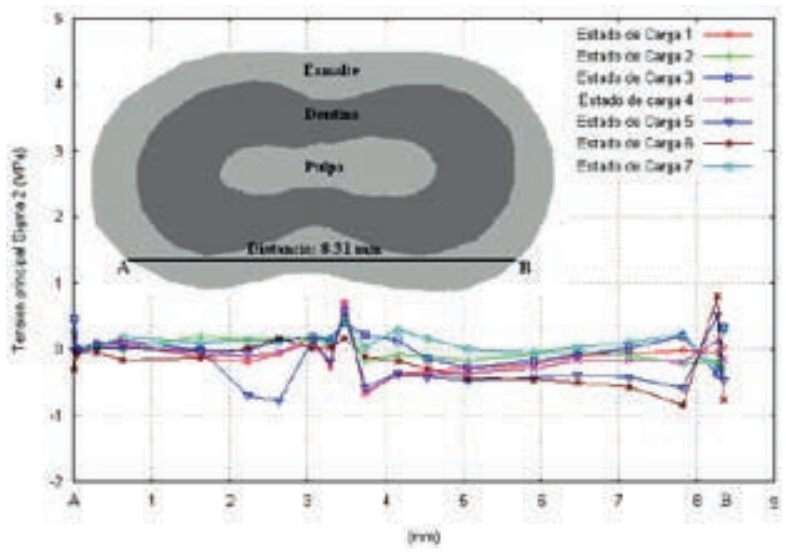

Figura 5. Distribución de tensión $\sigma_{2}$ a lo largo de la distancia AB. 


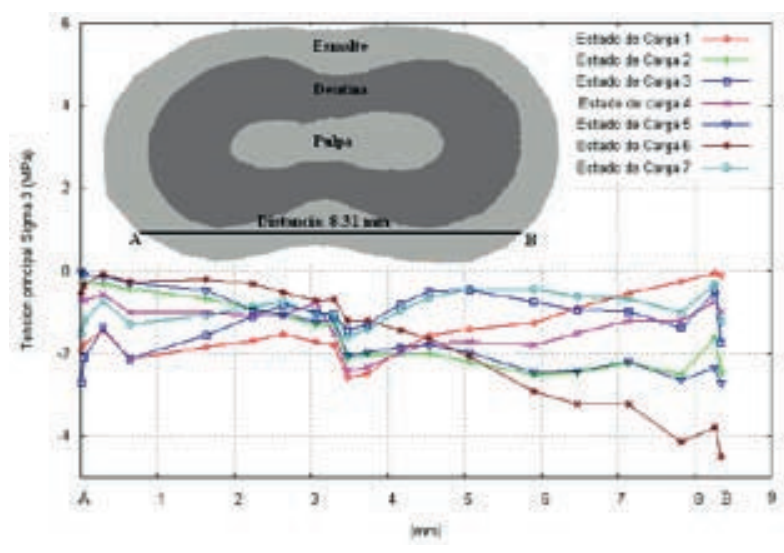

Figura 6. Distribución de tensión $\sigma_{3}$ (compresión) a lo largo de la distancia AB.

La figura 4 presenta la distribución de la tensión principal mayor $\sigma_{1}$ a lo largo de la recta $\mathrm{AB}$ sobre el esmalte dental. Como se aprecia, los estados de carga 1 y 4 generan una distribución de tensiones que tiende a desarrollar sus valores máximos a una distancia aproximada de $3.6 \mathrm{~mm}$ desde el punto A. Sin embargo, para los estados de carga 2, 3, 5, 6 y 7 los valores máximos tienden a concentrarse en la zona cercana al punto B ubicado sobre la cara vestibular. Cabe señalar, que las tensiones máximas de tracción $\sigma_{1}$ sobre el esmalte dental (punto B en figura 3) están asociadas fundamentalmente a cargas laterales, salvo el estado 5 que corresponde a una carga vertical sobre la cúspide bucal. Se aprecia que los estados de carga que inducen las mayores tensiones de tracción en la zona cervical corresponden al 6 y al 3, ambos asociados a cargas laterales.

La figura 5 presenta la distribución de la tensión principal intermedia $\sigma_{2}$ a lo largo de la recta $\mathrm{AB}$, tal como se muestra en dicha figura, esta tensión es prácticamente uniforme a lo largo de la recta $\mathrm{AB}$, salvo para el estado de carga 5 en donde se aprecia un aumento de la tensión a una distancia de aproximadamente de $3.6 \mathrm{~mm}$ del punto A, situación similar a lo ocurrido con $\sigma_{2}$ para los estados de carga 1 y 4 .

Finalmente la figura 6 muestra la distribución de la tensión principal menor $\sigma_{3}$ a lo largo de la recta $A B$, tal como aprecia en dicha figura esta tensión es de compresión alcanzando su valor máximo para estado de carga 6 en el punto B ubicado sobre la cara vestibular.

\section{CONCLUSIONES}

Los resultados obtenidos en la presente investigación son una evidencia biomecánica de lo propuesto por la teoría de abfracción [3], dado que numéricamente se demuestra que los esfuerzos máximos de tracción sobre el esmalte dental se concentran en la zona cervical de la cara vestibular y se encuentran asociados fundamentalmente a las cargas inclinadas. Las estados de carga críticos desde el punto de vista del nivel de tensiones de tracción sobre el esmalte dental en la zona cervical corresponden al 6 (carga bucal a $45^{\circ}$ del eje vertical en la cúspide bucal) y al 3 (carga lingual a $45^{\circ}$ del eje vertical en la cúspide lingual), para dichos estados de carga las tensiones de tracción sobre el esmalte dental son del orden de 2.5 a $3 \mathrm{MPa}$. Finalmente, se debe señalar que las zonas en donde se observan tensiones de tracción elevadas $\sigma_{1}$ concuerdan aproximadamente con las zonas en donde clínicamente se comprueban la ocurrencia de lesiones cervicales no cariosas.

\section{REFERENCIAS}

[1] D. Donetch. "Análisis de falla para lesiones cervicales no-cariosas en un primer premolar superior". Tesis para optar al título de Ingeniero Civil. Departamento de Ingeniería Civil. Facultad de Ingeniería. Universidad de Concepción, pp. 140. 2006.

[2] V.K. Goel, S.C. Khera, J.L. Ralston, K.H. Chang. "Stresses at the dentinoenamel junction of human teeth-a finite element investigation". Journal of Prosthetic Dentistry. Vol. 66, pp. 451-459. 1991.

[3] J.O. Grippo. "Abfractions. A new classification of hard tissue lesions of teeth". J. Esthet. Dent. Vol. 4, pp. 14-19. 1991.

[4] W.C. Lee, W.S. Eackle. "Possible role of tensile stress in the etiology of cervical erosive lesions of teeth". Journal of Prosthetic Dentistry. Vol. 52, pp. 374-380. 1984.

[5] H.E. Lee, C.L. Lin, C.H. Wang, C.H. Cheng, C.H. Chang. "Stresses at the cervical lesion of maxillary premolar - a finite element investigation". Journal of Dentistry. Vol. 30 No 7-8, pp. 283-290. 2002.

[6] C.E. Nemcovsky, Z. Artzi. "Erosion-abrasive lesions revisited”. Compendium. Vol. 17, pp. 416-423. 1996. 
[7] J.S. Rees, M. Hammadeh. "Undermining of enamel as a mechanism of abfraction lesion formation: a finite element study". European Journal of Oral Sciences. Vol. 112, pp. 347-352. August 2004.

[8] Samtech Headquaters. Liege science park. Rue des Chasseurs-Ardennais. 8 B-4031. Belgium.
February 2006. URLs: http://www.samcef. com

[9] L.G. Selna, HT. Shillingberg, PA. Kerr. "Finite element analysis of dental structures - axisymmetric and plane stress idealizations". Journal of Biomedical Material Research. Vol. 9, pp. 237-252.1975. 